\title{
Outflows and Turbulence in Young Stellar Clusters - An Observer's View
}

\author{
Héctor G. Arce \\ Department of Astronomy, Yale University, P.O. Box 208101, New Haven CT 06520 \\ email: hector.arce@yale.edu
}

\begin{abstract}
Recent numerical studies have focused their interest on the impact outflows have on the cloud's turbulence. The contradictory results obtained by these studies indicate that it is essential for observers to provide the required data to constrain the models. Here we discuss the impact of outflows on the environment surrounding clusters of young stellar objects, from an observer's point of view. We have conducted several studies of outflows in different active star-forming regions. In all cases it is clear that outflows have the power to sustain the observed turbulence in the gas surrounding protostellar clusters. We investigate whether there is a correlation between outflow strength and star formation efficiency, as predicted by numerical simulations, for six different regions in the Perseus molecular cloud complex. We argue that results of other recent studies that use CO line maps to study the turbulence driving length should not be used to discard outflows as major drivers of turbulence in clusters.
\end{abstract}

Keywords. stars: formation, stars: pre-main-sequence, ISM: jets and outflows, turbulence

\section{Introduction}

The outflowing supersonic wind from a protostar can accelerate the surrounding molecular gas to velocities significantly greater than those of the quiescent cloud gas thereby producing a molecular outflow that injects momentum and kinetic energy into its surroundings (Arce et al. 2007). Recent numerical studies show that outflows can couple strongly to the cloud and are highly efficient at driving turbulent motions (e.g., Carroll et al. 2009) and can also regulate the cloud's star formation efficiency (Nakamura \& Li 2007; Wang et al. 2010). Yet, other numerical studies suggest that protostellar outflows do a poor job at driving cloud turbulence, but can disrupt dense clumps and affect the cloud structure (Banerjee et al. 2007). These studies show the increased attention that research on the impact of outflows has obtained, as well as the need for targeted observations required to constrain the models and to reconcile their discrepancies.

Observational studies have shown that outflows, even from low-mass stars, can have an impact on their cloud at different distances from the source, ranging from a few thousand AU to several parsecs. Survey studies of the circumstellar gas within $10^{4}$ AU of low-mass protostars indicate outflows contribute significantly to the mass-loss of the surrounding dense gas (Fuller \& Ladd 2002; Arce \& Sargent 2006). An outflow's impact on its parent core, at distances of about 0.1 to $0.3 \mathrm{pc}$ from the forming star, is evidenced through the detection of outflow-blown cavities and outflowing dense gas (e.g., Tafalla \& Myers 1997). Giant outflows from young stars with sizes exceding $1 \mathrm{pc}$ in length are common (Reipurth et al. 1997). These outflows can interact with the surrounding medium and induce changes in the velocity and density distribution of the parent cloud's gas at large distances from the source (Arce \& Goodman 2001; 2002). Millimeter studies have shown that many molecular outflows produced by a group or cluster of young stars interact with a substantial volume of the cluster's environment, may sweep up the gas and dust and 
have the energy required for driving turbulence (e.g., Knee \& Sandell 2000). However, most of these studies concentrate on individual regions, making it difficult for a consistent comparison of the outflow impact in different clusters.

\section{Survey of Molecular Outflows in Perseus}

Recently, Arce et al. (2010, hereafter A10) conducted an unbiased outflow survey of the Perseus molecular cloud complex and were able to study how individual and groups of outflows effect the dynamics of the gas in the entire cloud complex and how they interact with their surroundings at different distances from the driving source (from about $0.1 \mathrm{pc}$ to a few parsecs). They discovered numerous new molecular outflow candidates in regions that had been poorly studied before (e.g., outskirts of famous star forming regions and regions with very low star formation activity). The new outflow candidates more than double the amount of outflow mass, momentum, and kinetic energy in the Perseus cloud complex compared to the total obtained from previously known outflows. Their results indicate that outflows have significant impact on the environment immediately surrounding localized regions of active star formation, but lack the energy needed to feed the observed turbulence in the entire Perseus complex.

As expected, outflows are concentrated in regions of high star formation activity, and it is in these regions (with sizes of 1 to $4 \mathrm{pc}$ ) that outflows have the biggest impact. In their study, A10 defined six regions of active star formation in the Perseus molecular cloud complex where clusters or groups of outflows were found (e.g., L1448, NGC 1333, B1-ridge, B1, IC 348, and B5). Their unbiased outflow search allowed them to study the impact of outflows in each region and to compare the different regions in a consistent manner. One way to assess the importance of outflows in driving the turbulence in their local environment is to compare the total outflow energy input rate into the cloud (i.e., outflow luminosity) with the energy rate needed to maintain the turbulence in the gas. For all six regions, the total outflow energy input rate into the molecular gas is at least $80 \%$ of the power needed to maintain the turbulence in the surrounding cluster gas. These results indicate that outflows in an active region of star formation can be a source of non-negligible power for driving turbulence in the molecular gas, and they should be treated as such in numerical simulations of star-forming clouds.

\section{Comparing Data with Numerical Simulations}

Recent numerical simulations have shown that the turbulence in an isolated clusterforming region fed by the energy and momentum injected by bipolar outflows can maintain the region close to (but not entirely in) dynamic equilibrium (Nakamura \& Li 2007; Wang et al. 2010). Simulations show that there is a small net flux of mass towards the bottom of the potential well that results in a slower collapse, compared to simulations without outflows, and only a few percent of the total gas mass is converted into stars within a free-fall time. These studies show that more powerful outflows result in an increase in the turbulence in the gas, which then lead to a delay of the gravitational collapse and, consequently, a lower star formation efficiency per free-fall time. In their study, A10 attempted to investigate whether there is any relationship between the star formation efficiency and the total outflow strength in the different star-forming regions in the Perseus cloud, and Figure 1 summarizes their results. This plot shows the normalized star formation efficiency $\left(S F E_{n}\right)$ as a function of $r_{L}$, the ratio of the total outflow mechanical luminosity in the region $\left(L_{\text {flow }}\right)$ to the energy rate needed to maintain the 


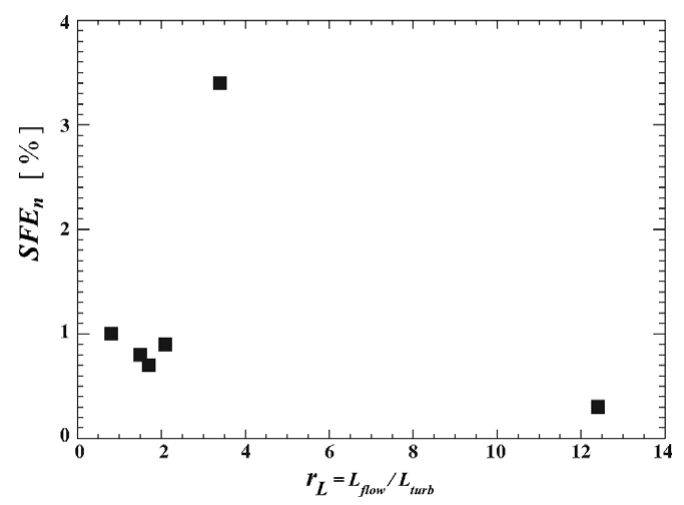

Figure 1. Normalized star formation efficiency as a function of $r_{L}$ for different regions of star formation in Perseus. No error bars are shown as it is hard to estimate accurate $1-\sigma$ uncertainties for these points. The uncertainties in the values of $S F E_{n}$ and $r_{L}$ could easily be a factor of two (or more). Figure from Arce et al. (2010)

turbulence in the gas $\left(L_{t u r b}\right)$. Here, $S F E_{n}$ is the star formation efficiency (SFE) multiplied by the ratio of the free-fall timescale of the region to the age of the region. This normalization is needed in order to take care of the fact that: 1) a cloud with a constant star formation rate will exhibit a higher star formation efficiency as the cloud evolves, since more of the cloud gas will be transformed into stars; and 2) regions with different free-fall times will collapse at different rates and thus will exhibit different values of the current SFE, even for clouds of the same age (see A10 for details). We use $r_{L}$ as a way to quantify the outflow strength and their impact on the cloud's turbulence. Clouds with $r_{L}>1$ harbor outflows that input enough energy into the surrounding gas to maintain the observed turbulence in the cloud. Hence, we would expect clouds with a high $r_{L}$ to have a low $S F E_{n}$.

Figure 1 does not show a clear dependence of $S F E_{n}$ with $r_{L}$. We see that the region with the largest $r_{L}$ exhibits the lowest value of $S F E_{n}$, but otherwise there is no correlation between $r_{L}$ and $S F E_{n}$. It is hard to draw any strong conclusion from these results. Given the large uncertainties in $S F E_{n}$, the small size of the sample and the limited range in values of the total outflow momentum and $r_{L}$ among all regions, it is of no surprise that we do not find a significant correlation between $S F E_{n}$ and outflow strength, even if such correlation exists. Clearly, similar additional studies are needed to increase the number of star-forming regions and eventually obtain a statistically sound sample of clusters with outflows.

Other recent studies have claimed that even in a region full of outflows like NGC 1333, outflows are not the main source of turbulence in the gas (Brunt et al. 2009; Padoan et al. 2009). The results of the analysis of the gas kinematics of these two studies, using a ${ }^{13} \mathrm{CO}(1-0)$ map of the region, suggest that turbulence is mostly driven at scales larger than the region of interest. They claim that outflows are not the main drivers of the region's turbulence because if that were the case the velocity power spectrum would show clear evidence that the turbulence is being driven at a scale of approximately 0.3 pc. We argue that in clusters outflow-driven turbulence should not necessarily exhibit a driving scale close to $0.3 \mathrm{pc}$. One possibility is that outflows drive turbulence at a very small scale (about 0.05 pc), as suggested by Swift \& Welch (2008) - a scale that would not have been resolved by the data used by Brunt et al. (2009) and Padoan et al. (2009). Alternatively, the fact that outflows exhibit a range of sizes from less than $0.1 \mathrm{pc}$ to more than a few parsecs in length may result in outflows driving turbulence at different 


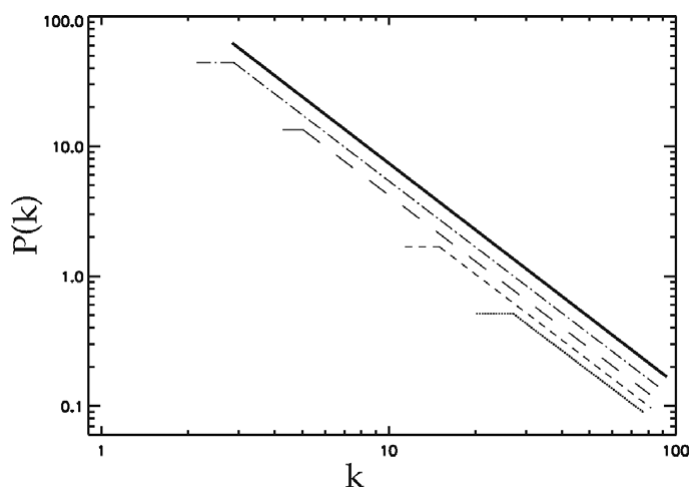

Figure 2. Schematic representation of turbulence power spectrum $(P)$ as a function of wavenumber $(k)$. Different lines represent the expected power spectrum for turbulence driven by outflows at scales of $0.3 \mathrm{pc}$ (dotted), $0.5 \mathrm{pc}$ (small dash), $1.5 \mathrm{pc}$ (long dash), $2.5 \mathrm{pc}$ (dash-dot). The solid line represents the resultant (total) observed power spectrum for a region with outflows that drive turbulence at all the scales listed above.

size scales. As a consequence we should not expect outflow-driven turbulence in a cluster (with many outflows of different sizes and from different sources) to exhibit clear evidence of being driven at a specific scale. In fact, given that outflows may extent to sizes of a few parsecs, they can drive turbulence all the way at scales similar to the size scales of the region, mimicking the expected behavior of the velocity power spectrum (as a function of scale) of turbulence driven by sources external to the cloud. A schematic picture of this is depicted in Figure 2, where the velocity power spectrum as a function of wavenumber (approximately the reciprocal of the size scale) is shown for turbulence driven at different scales. It can be seen that if outflows drive turbulence at different scales, then the shape of the resulting (total) power spectrum will be similar to the power spectrum of turbulence driven at the largest scales. Hence, the results of these recent studies cannot be used to reject the idea that outflows may be a major source of turbulence in clusters.

\section{References}

Arce, H. G. \& Goodman, A. A. 2001, ApJ, 554, 132

Arce, H. G. \& Goodman, A. A. 2002, ApJ, 575, 911

Arce, H. G. \& Sargent, A. I. 2006, ApJ, 646, 1070

Arce, H. G., Shepherd, D., Gueth, F., Lee, C.-F., Bachiller, R., Rosen, A., \& Beuther, H. 2007, in Protostars and Planets V, eds. B. Reipurth, D. Jewitt, and K. Keil, (University of Arizona Press: Tucson), 245

Arce, H. G.; Borkin, M. A., Goodman, A. A., Pineda, J. E., \& Halle, M. 2010, ApJ, 715, 1170

Banerjee, R., Klessen, R. S., \& Fendt, C. 2007, ApJ, 668, 1028

Brunt, C. M., Heyer, M. H., \& Mac Low, M.-M. 2009, A\&SA, 504, 883

Carroll, J., Frank, A., Blackman, E., Cunningham, A., \& Quillen, A. 2009, ApJ, 695, 1376

Fuller, G. A. \& Ladd, E. F. 2002, ApJ, 573, 699

Knee, L. B. G. \& Sandell, G. 2000, A\& $A$, 361, 671

Nakamura, F. \& Li, Z.-Y. 2007, ApJ, 662, 395

Padoan, P., Juvela, M., Kritsuk, A., \& Norman, M. L. 2009, ApJ, 707, L153

Reipurth, B., Bally, J., \& Devine, D. 1997, AJ, 114, 2708

Swift, J. J. \& Welch, W. J. 2008, ApJS, 174, 202

Tafalla, M. \& Myers, P. C. 1997, ApJ, 491, 653

Wang, P., Li, Z-.Y., Abel, T., \& Nakamura, F. 2010, ApJ, 709, 27 